\title{
Cell Petri Net Concepts
}

\author{
Mitsuru Jitsukawa \\ Chiba-ken Asahi-shi Kotoda 2927-13 \\ 289-2502 Japan \\ Yasunari Shidama
Shinshu University
Nagano, Japan
}

\author{
Pauline N. Kawamoto \\ Shinshu University \\ Nagano, Japan
}

\author{
Yatsuka Nakamura \\ Shinshu University \\ Nagano, Japan
}

\begin{abstract}
Summary. Based on the Petri net definitions and theorems already formalized in [8], with this article, we developed the concept of "Cell Petri Nets". It is based on [9]. In a cell Petri net we introduce the notions of colors and colored states of a Petri net, connecting mappings for linking two Petri nets, firing rules for transitions, and the synthesis of two or more Petri nets.
\end{abstract}

MML identifier: PETRI_2, version: $\underline{7.11 .014 .117 .1046}$

The papers [11], [12], [6], [13], [14], [10], [8], [2], [5], [3], [4], [7], and [1] provide the terminology and notation for this paper.

\section{Preliminaries: Thin Cylinder, Locus}

Let $A$ be a non empty set, let $B$ be a set, let $B_{1}$ be a set, and let $y_{1}$ be a function from $B_{1}$ into $A$. Let us assume that $B_{1} \subseteq B$. The functor cylinder $_{0}\left(A, B, B_{1}, y_{1}\right)$ yields a non empty subset of $A^{B}$ and is defined by:

(Def. 1) $\operatorname{cylinder}_{0}\left(A, B, B_{1}, y_{1}\right)=\left\{y: B \rightarrow A: y\left\lceil B_{1}=y_{1}\right\}\right.$.

Let $A$ be a non empty set and let $B$ be a set. A non empty subset of $A^{B}$ is said to be a thin cylinder of $A$ and $B$ if:

(Def. 2) There exists a subset $B_{1}$ of $B$ and there exists a function $y_{1}$ from $B_{1}$ into $A$ such that $B_{1}$ is finite and it $=\operatorname{cylinder}_{0}\left(A, B, B_{1}, y_{1}\right)$.

The following propositions are true: 
(1) Let $A$ be a non empty set, $B$ be a set, and $D$ be a thin cylinder of $A$ and $B$. Then there exists a subset $B_{1}$ of $B$ and there exists a function $y_{1}$ from $B_{1}$ into $A$ such that $B_{1}$ is finite and $D=\left\{y: B \rightarrow A: y\left\lceil B_{1}=y_{1}\right\}\right.$.

(2) Let $A_{1}, A_{2}$ be non empty sets, $B$ be a set, and $D_{1}$ be a thin cylinder of $A_{1}$ and $B$. If $A_{1} \subseteq A_{2}$, then there exists a thin cylinder $D_{2}$ of $A_{2}$ and $B$ such that $D_{1} \subseteq D_{2}$.

Let $A$ be a non empty set and let $B$ be a set. The thin cylinders of $A$ and $B$ constitute a non empty family of subsets of $A^{B}$ defined by:

(Def. 3) The thin cylinders of $A$ and $B=\left\{D \subseteq A^{B}: D\right.$ is a thin cylinder of $A$ and $B$ \}.

We now state three propositions:

(3) Let $A$ be a non trivial set, $B$ be a set, $B_{2}$ be a set, $y_{2}$ be a function from $B_{2}$ into $A, B_{3}$ be a set, and $y_{3}$ be a function from $B_{3}$ into $A$. If $B_{2} \subseteq B$ and $B_{3} \subseteq B$ and $\operatorname{cylinder}_{0}\left(A, B, B_{2}, y_{2}\right)=\operatorname{cylinder}_{0}\left(A, B, B_{3}, y_{3}\right)$, then $B_{2}=B_{3}$ and $y_{2}=y_{3}$.

(4) Let $A_{1}, A_{2}$ be non empty sets and $B_{4}, B_{5}$ be sets. Suppose $A_{1} \subseteq A_{2}$ and $B_{4} \subseteq B_{5}$. Then there exists a function $F$ from the thin cylinders of $A_{1}$ and $B_{4}$ into the thin cylinders of $A_{2}$ and $B_{5}$ such that for every set $x$ if $x \in$ the thin cylinders of $A_{1}$ and $B_{4}$, then there exists a subset $B_{1}$ of $B_{4}$ and there exists a function $y_{2}$ from $B_{1}$ into $A_{1}$ and there exists a function $y_{3}$ from $B_{1}$ into $A_{2}$ such that $B_{1}$ is finite and $y_{2}=y_{3}$ and $x=\operatorname{cylinder}_{0}\left(A_{1}, B_{4}, B_{1}, y_{2}\right)$ and $F(x)=$ cylinder $_{0}\left(A_{2}, B_{5}, B_{1}, y_{3}\right)$.

(5) Let $A_{1}, A_{2}$ be non empty sets and $B_{4}, B_{5}$ be sets. Then there exists a function $G$ from the thin cylinders of $A_{2}$ and $B_{5}$ into the thin cylinders of $A_{1}$ and $B_{4}$ such that for every set $x$ if $x \in$ the thin cylinders of $A_{2}$ and $B_{5}$, then there exists a subset $B_{3}$ of $B_{5}$ and there exists a subset $B_{2}$ of $B_{4}$ and there exists a function $y_{2}$ from $B_{2}$ into $A_{1}$ and there exists a function $y_{3}$ from $B_{3}$ into $A_{2}$ such that $B_{2}$ is finite and $B_{3}$ is finite and $B_{2}=B_{4} \cap B_{3} \cap y_{3}{ }^{-1}\left(A_{1}\right)$ and $y_{2}=y_{3}\left\lceil B_{2}\right.$ and $x=\operatorname{cylinder}_{0}\left(A_{2}, B_{5}, B_{3}, y_{3}\right)$ and $G(x)=$ cylinder $_{0}\left(A_{1}, B_{4}, B_{2}, y_{2}\right)$.

Let $A_{1}, A_{2}$ be non trivial sets and let $B_{4}, B_{5}$ be sets. Let us assume that there exist sets $x, y$ such that $x \neq y$ and $x, y \in A_{1}$ and $A_{1} \subseteq A_{2}$ and $B_{4} \subseteq B_{5}$. The functor Extcylinders $\left(A_{1}, B_{4}, A_{2}, B_{5}\right)$ yielding a function from the thin cylinders of $A_{1}$ and $B_{4}$ into the thin cylinders of $A_{2}$ and $B_{5}$ is defined by the condition (Def. 4).

(Def. 4) Let $x$ be a set. Suppose $x \in$ the thin cylinders of $A_{1}$ and $B_{4}$. Then there exists a subset $B_{1}$ of $B_{4}$ and there exists a function $y_{2}$ from $B_{1}$ into $A_{1}$ and there exists a function $y_{3}$ from $B_{1}$ into $A_{2}$ such that $B_{1}$ is finite and $y_{2}=y_{3}$ and $x=\operatorname{cylinder}_{0}\left(A_{1}, B_{4}, B_{1}, y_{2}\right)$ and $\left(\operatorname{Extcylinders}\left(A_{1}, B_{4}, A_{2}, B_{5}\right)\right)(x)=$ cylinder $_{0}\left(A_{2}, B_{5}, B_{1}, y_{3}\right)$. 
Let $A_{1}$ be a non empty set, let $A_{2}$ be a non trivial set, and let $B_{4}$, $B_{5}$ be sets. Let us assume that $A_{1} \subseteq A_{2}$ and $B_{4} \subseteq B_{5}$. The functor Ristcylinders $\left(A_{1}, B_{4}, A_{2}, B_{5}\right)$ yields a function from the thin cylinders of $A_{2}$ and $B_{5}$ into the thin cylinders of $A_{1}$ and $B_{4}$ and is defined by the condition (Def. 5).

(Def. 5) Let $x$ be a set. Suppose $x \in$ the thin cylinders of $A_{2}$ and $B_{5}$. Then there exists a subset $B_{3}$ of $B_{5}$ and there exists a subset $B_{2}$ of $B_{4}$ and there exists a function $y_{2}$ from $B_{2}$ into $A_{1}$ and there exists a function $y_{3}$ from $B_{3}$ into $A_{2}$ such that $B_{2}$ is finite and $B_{3}$ is finite and $B_{2}=$ $B_{4} \cap B_{3} \cap y_{3}{ }^{-1}\left(A_{1}\right)$ and $y_{2}=y_{3}\left\lceil B_{2}\right.$ and $x=\operatorname{cylinder}_{0}\left(A_{2}, B_{5}, B_{3}, y_{3}\right)$ and $\left(\right.$ Ristcylinders $\left.\left(A_{1}, B_{4}, A_{2}, B_{5}\right)\right)(x)=\operatorname{cylinder}_{0}\left(A_{1}, B_{4}, B_{2}, y_{2}\right)$.

Let $A$ be a non trivial set, let $B$ be a set, and let $D$ be a thin cylinder of $A$ and $B$. The functor loc $D$ yielding a finite subset of $B$ is defined by the condition (Def. 6).

(Def. 6) There exists a subset $B_{1}$ of $B$ and there exists a function $y_{1}$ from $B_{1}$ into $A$ such that $B_{1}$ is finite and $D=\left\{y: B \rightarrow A: y\left\lceil B_{1}=y_{1}\right\}\right.$ and $\operatorname{loc} D=B_{1}$.

\section{Colored Petri Nets}

Let $A_{1}, A_{2}$ be non trivial sets, let $B_{4}, B_{5}$ be sets, let $C_{1}, C_{2}$ be non trivial sets, let $D_{1}, D_{2}$ be sets, and let $F$ be a function from the thin cylinders of $A_{1}$ and $B_{4}$ into the thin cylinders of $C_{1}$ and $D_{1}$. The functor CylinderFunc $\left(A_{1}, B_{4}, A_{2}, B_{5}, C_{1}, D_{1}, C_{2}, D_{2}, F\right)$ yielding a function from the thin cylinders of $A_{2}$ and $B_{5}$ into the thin cylinders of $C_{2}$ and $D_{2}$ is defined as follows:

(Def. 7) CylinderFunc $\left(A_{1}, B_{4}, A_{2}, B_{5}, C_{1}, D_{1}, C_{2}, D_{2}, F\right)=$ Extcylinders $\left(C_{1}, D_{1}, C_{2}, D_{2}\right) \cdot F \cdot$ Ristcylinders $\left(A_{1}, B_{4}, A_{2}, B_{5}\right)$.

We consider colored place/transition net structures as extensions of place/transition net structure as systems

$\langle$ places, transitions, S-T arcs, T-S arcs, a colored set, a firing-rule $\rangle$, where the places and the transitions constitute non empty sets, the S-T arcs constitute a non empty relation between the places and the transitions, the T-S arcs constitute a non empty relation between the transitions and the places, the colored set is a non empty finite set, and the firing-rule is a function.

Let $C_{3}$ be a colored place/transition net structure and let $t_{0}$ be a transition of $C_{3}$. We say that $t_{0}$ is outbound if and only if:

(Def. 8) $\overline{\left\{t_{0}\right\}}=\emptyset$.

Let $C_{4}$ be a colored place/transition net structure. The functor Outbds $C_{4}$ yielding a subset of the transitions of $C_{4}$ is defined by:

(Def. 9) Outbds $C_{4}=\left\{x ; x\right.$ ranges over transitions of $C_{4}: x$ is outbound $\}$. 
Let $C_{3}$ be a colored place/transition net structure. We say that $C_{3}$ is coloredPT-net-like if and only if the conditions (Def. 10) are satisfied.

(Def. 10)(i) $\operatorname{dom}$ (the firing-rule of $\left.C_{3}\right) \subseteq$ (the transitions of $C_{3}$ ) \Outbds $C_{3}$, and

(ii) for every transition $t$ of $C_{3}$ such that $t \in \operatorname{dom}$ (the firing-rule of $C_{3}$ ) there exists a non empty subset $C_{5}$ of the colored set of $C_{3}$ and there exists a subset $I$ of $*\{t\}$ and there exists a subset $O$ of $\overline{\{t\}}$ such that (the firing-rule of $\left.C_{3}\right)(t)$ is a function from the thin cylinders of $C_{5}$ and $I$ into the thin cylinders of $C_{5}$ and $O$.

We now state two propositions:

(6) Let $C_{3}$ be a colored place/transition net structure and $t$ be a transition of $C_{3}$. Suppose $C_{3}$ is colored-PT-net-like and $t \in \operatorname{dom}$ (the firing-rule of $C_{3}$ ). Then there exists a non empty subset $C_{5}$ of the colored set of $C_{3}$ and there exists a subset $I$ of ${ }^{*}\{t\}$ and there exists a subset $O$ of $\overline{\{t\}}$ such that (the firing-rule of $\left.C_{3}\right)(t)$ is a function from the thin cylinders of $C_{5}$ and $I$ into the thin cylinders of $C_{5}$ and $O$.

(7) Let $C_{4}, C_{6}$ be colored place/transition net structures, $t_{1}$ be a transition of $C_{4}$, and $t_{2}$ be a transition of $C_{6}$. Suppose that

(i) the places of $C_{4} \subseteq$ the places of $C_{6}$,

(ii) the transitions of $C_{4} \subseteq$ the transitions of $C_{6}$,

(iii) the S-T $\operatorname{arcs}$ of $C_{4} \subseteq$ the $\mathrm{S}-\mathrm{T}$ arcs of $C_{6}$,

(iv) the T-S arcs of $C_{4} \subseteq$ the T-S arcs of $C_{6}$, and

(v) $t_{1}=t_{2}$.

Then $*\left\{t_{1}\right\} \subseteq *\left\{t_{2}\right\}$ and $\overline{\left\{t_{1}\right\}} \subseteq \overline{\left\{t_{2}\right\}}$.

One can verify that there exists a colored place/transition net structure which is strict and colored-PT-net-like.

A colored place/transition net is a colored-PT-net-like colored place/transition net structure.

\section{Color Counts of CPNT}

Let $C_{4}, C_{6}$ be colored place/transition net structures. We say that $C_{4}$ misses $C_{6}$ if and only if:

(Def. 11) (The places of $\left.C_{4}\right) \cap$ (the places of $\left.C_{6}\right)=\emptyset$ and (the transitions of $\left.C_{4}\right) \cap\left(\right.$ the transitions of $\left.C_{6}\right)=\emptyset$.

Let us note that the predicate $C_{4}$ misses $C_{6}$ is symmetric.

\section{Colored States of CPNT}

Let $C_{4}$ be a colored place/transition net structure and let $C_{6}$ be a colored place/transition net structure. Connecting mapping of $C_{4}$ and $C_{6}$ is defined by the condition (Def. 12). 
(Def. 12) There exists a function $O_{12}$ from Outbds $C_{4}$ into the places of $C_{6}$ and there exists a function $O_{21}$ from Outbds $C_{6}$ into the places of $C_{4}$ such that it $=\left\langle O_{12}, O_{21}\right\rangle$.

\section{Outbound Transitions of CPNT}

Let $C_{4}, C_{6}$ be colored place/transition nets and let $O$ be a connecting mapping of $C_{4}$ and $C_{6}$. Connecting firing rule of $C_{4}, C_{6}$, and $O$ is defined by the condition (Def. 13).

(Def. 13) There exist functions $q_{12}, q_{21}$ and there exists a function $O_{12}$ from Outbds $C_{4}$ into the places of $C_{6}$ and there exists a function $O_{21}$ from Outbds $C_{6}$ into the places of $C_{4}$ such that

(i) $O=\left\langle O_{12}, O_{21}\right\rangle$,

(ii) $\operatorname{dom} q_{12}=$ Outbds $C_{4}$,

(iii) $\operatorname{dom} q_{21}=$ Outbds $C_{6}$,

(iv) for every transition $t_{3}$ of $C_{4}$ such that $t_{3}$ is outbound holds $q_{12}\left(t_{3}\right)$ is a function from the thin cylinders of the colored set of $C_{4}$ and $*\left\{t_{3}\right\}$ into the thin cylinders of the colored set of $C_{4}$ and $O_{12}{ }^{\circ} t_{3}$,

(v) for every transition $t_{4}$ of $C_{6}$ such that $t_{4}$ is outbound holds $q_{21}\left(t_{4}\right)$ is a function from the thin cylinders of the colored set of $C_{6}$ and $*\left\{t_{4}\right\}$ into the thin cylinders of the colored set of $C_{6}$ and $O_{21}{ }^{\circ} t_{4}$, and

(vi) it $=\left\langle q_{12}, q_{21}\right\rangle$.

\section{Connecting Mapping for CPNT1, CPNT2}

Let $C_{4}, C_{6}$ be colored place/transition nets, let $O$ be a connecting mapping of $C_{4}$ and $C_{6}$, and let $q$ be a connecting firing rule of $C_{4}, C_{6}$, and $O$. Let us assume that $C_{4}$ misses $C_{6}$. The functor synthesis $\left(C_{4}, C_{6}, O, q\right)$ yielding a strict colored place/transition net is defined by the condition (Def. 14).

(Def. 14) There exist functions $q_{12}, q_{21}$ and there exists a function $O_{12}$ from Outbds $C_{4}$ into the places of $C_{6}$ and there exists a function $O_{21}$ from Outbds $C_{6}$ into the places of $C_{4}$ such that

$O=\left\langle O_{12}, O_{21}\right\rangle$ and $\operatorname{dom} q_{12}=$ Outbds $C_{4}$ and $\operatorname{dom} q_{21}=$ Outbds $C_{6}$ and for every transition $t_{3}$ of $C_{4}$ such that $t_{3}$ is outbound holds $q_{12}\left(t_{3}\right)$ is a function from the thin cylinders of the colored set of $C_{4}$ and ${ }^{*}\left\{t_{3}\right\}$ into the thin cylinders of the colored set of $C_{4}$ and $O_{12}{ }^{\circ} t_{3}$ and for every transition $t_{4}$ of $C_{6}$ such that $t_{4}$ is outbound holds $q_{21}\left(t_{4}\right)$ is a function from the thin cylinders of the colored set of $C_{6}$ and $*\left\{t_{4}\right\}$ into the thin cylinders of the colored set of $C_{6}$ and $O_{21}{ }^{\circ} t_{4}$ and $q=\left\langle q_{12}, q_{21}\right\rangle$ and the places of synthesis $\left(C_{4}, C_{6}, O, q\right)=\left(\right.$ the places of $\left.C_{4}\right) \cup\left(\right.$ the places of $\left.C_{6}\right)$ and the 
transitions of synthesis $\left(C_{4}, C_{6}, O, q\right)=\left(\right.$ the transitions of $\left.C_{4}\right) \cup$ (the transitions of $\left.C_{6}\right)$ and the S-T arcs of synthesis $\left(C_{4}, C_{6}, O, q\right)=($ the S-T $\operatorname{arcs}$ of $\left.C_{4}\right) \cup\left(\right.$ the S-T $\operatorname{arcs}$ of $\left.C_{6}\right)$ and the T-S arcs of synthesis $\left(C_{4}, C_{6}, O, q\right)=($ the T-S arcs of $\left.C_{4}\right) \cup\left(\right.$ the T-S arcs of $\left.C_{6}\right) \cup O_{12} \cup O_{21}$ and the colored set of synthesis $\left(C_{4}, C_{6}, O, q\right)=\left(\right.$ the colored set of $\left.C_{4}\right) \cup\left(\right.$ the colored set of $\left.C_{6}\right)$ and the firing-rule of synthesis $\left(C_{4}, C_{6}, O, q\right)=$ (the firing-rule of $\left.C_{4}\right)+\cdot$ (the firing-rule of $\left.C_{6}\right)+\cdot q_{12}+\cdot q_{21}$.

\section{REFERENCES}

[1] Józef Białas. Group and field definitions. Formalized Mathematics, 1(3):433-439, 1990.

[2] Czesław Byliński. Functions and their basic properties. Formalized Mathematics, 1(1):5565, 1990.

[3] Czesław Byliński. Functions from a set to a set. Formalized Mathematics, 1(1):153-164, 1990.

[4] Czesław Byliński. The modification of a function by a function and the iteration of the composition of a function. Formalized Mathematics, 1(3):521-527, 1990.

[5] Czesław Byliński. Partial functions. Formalized Mathematics, 1(2):357-367, 1990.

[6] Czesław Byliński. Some basic properties of sets. Formalized Mathematics, 1(1):47-53, 1990.

[7] Agata Darmochwał. Finite sets. Formalized Mathematics, 1(1):165-167, 1990.

[8] Pauline N. Kawamoto, Yasushi Fuwa, and Yatsuka Nakamura. Basic Petri net concepts. Formalized Mathematics, 3(2):183-187, 1992.

[9] Pauline N. Kawamoto and Yatsuka Nakamura. On Cell Petri Nets. Journal of Applied Functional Analysis, 1996.

[10] Andrzej Trybulec. Domains and their Cartesian products. Formalized Mathematics, 1(1):115-122, 1990.

[11] Andrzej Trybulec. Enumerated sets. Formalized Mathematics, 1(1):25-34, 1990.

[12] Zinaida Trybulec. Properties of subsets. Formalized Mathematics, 1(1):67-71, 1990.

[13] Edmund Woronowicz. Relations and their basic properties. Formalized Mathematics, 1(1):73-83, 1990.

[14] Edmund Woronowicz. Relations defined on sets. Formalized Mathematics, 1(1):181-186, 1990. 